\title{
A Model Based Safety Architecture Framework for Dutch High Speed Train Lines
}

\author{
Schuitemaker K. \\ Design, Production and \\ Management Department \\ University of Twente \\ Enschede, the Netherlands \\ katja.schuitemaker@gmail.com
}

\author{
Braakhuis J.G. \\ Systems Engineering Department \\ Aircraft, Development and \\ Systems Engineering B.V. \\ Hoofddorp, the Netherlands
}

\author{
Rajabalinejad M. \\ Faculty of Engineering \\ Technology \\ University of Twente \\ Enschede, the Netherlands
}

\begin{abstract}
This paper presents a model-based safety architecture framework (MBSAF) for capturing and sharing architectural knowledge of safety cases of safetycritical systems of systems (SoS). Whilst architecture frameworks in the systems engineering domain consider safety often as dependent attribute, this study focusses specifically on sharing architectural knowledge of safety cases between stakeholders and managing safety in systems development. For this purpose, we adapt the A3 architecture overview (A3AO) tool. The application is shown though the case study of Dutch high speed train lines and shows how to derive requirements from various stakeholders by carrying out iterative validations of the A3AOs. The implemented technique consists of systems modeling language-based (SysML) diagrams. Outcomes of the assessment lead to guidelines for two A3AOs. This results in increasing and effective interaction between stakeholders, more overview for managing safety complexity, more insight into finding required safety information, and therefore; an increasing efficiency in safety engineering.
\end{abstract}

Keywords: SoSE, safety, architecture, framework, Model based

\section{Introduction}

The increasing complexity and interdisciplinarity of emerging Systems of Systems (SoS) -whose failure may cause injury or death to human beings- has resulted in a shift from document-based systems engineering to modelbased architecture frameworks. These frameworks manage system complexity, obtain system overview, encourage knowledge sharing, finding required system information, and enhance communication across disciplines and departments.

Additionally, the rising number of safety-critical SoS goes along with a shift to transparency and a rising need of the general public for safety.
In contrast, architecture frameworks in the systems engineering domain consider safety often as dependent attribute (A3AO, DoDAF, TOGAF) [1], [2], [3], and put safety more in the background, which could result in an incomplete safety analysis and therefore, an unsafe SoS. However, in software domain some frameworks have emerged that are primarily oriented on safety [4], [5], [6], [7], but they are not specifically focusing on sharing architectural knowledge, which is an essential factor for conducting a safety analysis of a large, complex and interdisciplinary SoS. A suitable approach to conduct the safety analysis for a safety-critical SoS which has become popular recently, is the safety case approach. It conducts an argument for why the system is safe by means of claims, evidence, arguments and inference rules.

In order to deal with these challenges and impacts on performance and security which are also described by Chiprianov et al [8] this work attempts to redress the gap between the need to improve knowledge sharing during the establishment of a safety case and the missing centralization of safety in any architecture framework in the systems engineering domain.

For this purpose, we want to focus specifically on enhancing communication across disciplines by improving the interaction between stakeholders to shorten the development cycle in setting up a safety case and therefore; increasing the efficiency in safety engineering. For this purpose, we adjust the A3 architecture overview (A3AO) tool described by Borches [1] to make it applicable to safety cases. Studies show that this approach is well perceived by users and successfully applied in many practices [1, chapter 11], [9], [10]. It focusses on capturing and sharing architectural knowledge by making implicit knowledge explicit. In addition, in order to reach a large group of stakeholders, we use the systems modeling language (SysML) [11], due to its increased use in industry.

In the first place, centralization of a safety case by allocating A3AOs to relevant meetings fosters focus and avoids idleness of stakeholders.

Second, modification of the A3AO tool by allocating functions extracted from safety standards to one of the three 
views, provides insight and facilitates finding required safety information and results in a collaboration and communication tool that provides support for managing safety complexity and safety overview.

Besides this, the derivation of requirements from users results in safety case-specific A3AOs which enhances acceptation and effectiveness of the architecture framework.

Last, guidelines provide a consistency in presenting architectural knowledge, making the framework reusable and contributing to recognition of the A3AOs.

Given these points, if we compare this model-based safety architecture framework (MBSAF) with traditional documents, the MBSAF captures and shares architectural knowledge of a safety case in A3AOs which results in increasing and effective interaction between stakeholders, more overview for managing safety complexity, more insight into finding required safety information, and therefore; an increasing efficiency in safety engineering.

\section{Background}

\subsection{SoS under study}

The SoS under study (here we refer to it as the safety case) considers a Dutch railway safety-critical SoS: the HSL-Zuid (Dutch: Hogesnelheidslijn Zuid, English: HighSpeed Line South), which is a complex SoS project with multiple stakeholders who sometimes have contrary interests. The problems of this SoS fall into the category: ill-structured problems [12]. It is set up according to recommendations outlined in EN50126 (railway applications - specification and demonstration of reliability, availability, maintainability and safety for railway applications) and EN50129 (railway applications communication, signaling and processing systems - safety related electronic systems for signaling). The safety analysis used is a Functional Hazard Analysis (FHA) which has as an effect that functions outlined in these safety standards are more of a functional nature. They are discussed in various meetings:

- Meeting on system definition:

Function 1. Define functional system: analyze system

- Meeting on hazard analysis:

Function 2. Identify functional hazards: analyze hazards and estimate hazard rates

Function 3. Analyze functional consequences: Forecast accidents

Function 4. Estimate risks: determine individual risk Function 5. Allocate tolerable hazard rates: defining integrity requirements

- Meeting on risk reduction:
Function 6. Control functional hazards: deliver barriers for prevention or contingency.

The HSL-Zuid safety case has been set up according to these functions, with a few adjustments:

- Function 2 is preceded by defining roles and responsibilities of the stakeholders for physical components.

- Function 2 and -3 are combined in the FHA, from which a graphical representation of the data model is shown in Figure 1.

- Function 6 is followed by an assessment of the barriers that control the functional hazards.

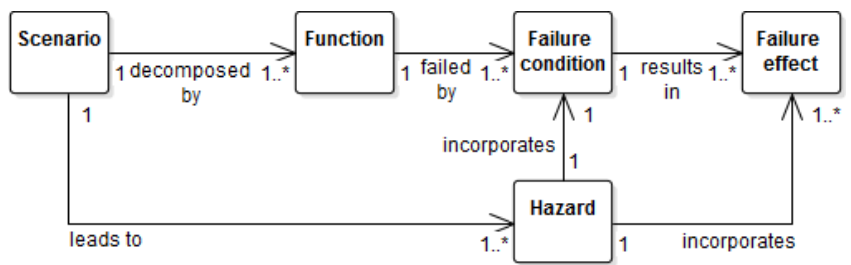

Figure 1. Data model of the FHA of the HSL-Zuid

\subsection{A3 architecture overviews tool}

An architecture framework aims to provide guidance for what information, what presentation and what structure to use to capture the architecture according to Zachman [13]. As the safety case approach requires continuous analyses as well as communication between stakeholders with various backgrounds, architecture frameworks can be of great value.

With this in mind, the incapability of stakeholders to cope with the number of views leads us to the A3 architecture overview (A3AO) tool. This approach is based on the use of only three views visualized on one A3-sheet:

- Functional view: behavioral view of functions, visualized as a functional flow.

- Physical view: physical breakdown, visualized as a building block view.

- Quantification view: key characteristics, visualized in textual form or through tables or graphs.

Next to these three views, the A3AO tool also makes use of:

- Visual aids: small visualizations to help understand the functions.

- Constraints and choices: notes that give information about constraints and choices that are made to establish this overview.

- Legend: explanation about the meanings of elements of that overview.

- Textual information on the back of the A3-sheet. 


\subsection{Bow-tie approach}

The proposed methodology for the functional view of one of is based on the bow-tie approach [14], which gained popularity [15], because of the simple overview of the different accident scenarios under analysis. A bow-tie diagram is represented by a top-event that can be considered as the accident, a list of potential threats on the left side of the top-event, and a list of the consequences on the right side of the top-event. It puts focus on the creation of barriers for discussing preventive (on the left-side) and corrective (on the right-side) elements.

\section{Method}

This study uses the well-known "Vee" process model, from which we use the methodology described by Blanchard and Fabrycky [16]. Requirements regarding the MBSAF are extracted from two sources: 1) Safety standards for architecting a complete safety case and; 2) Stakeholders for case-specific effectiveness.

At first, we extract functions from safety standards so that the goals of the three main views of the $\mathrm{A} 3 \mathrm{AO}$ tool will be adjusted.

Hereafter, we perform a case study to derive requirements from various stakeholders. The participants consist of 9 stakeholders with backgrounds varying from safety engineers, domain experts and other stakeholders who were all involved in the process of the set-up of the safety case HSL-Zuid. The derived requirements are based on evaluations of stakeholders regarding interaction improvement by various diagrams that are set up during this study and finishes with 4 user-validations of the MBSAF.

\section{Results}

\subsection{Allocation to meetings}

It is important to make a clear distinction between the meetings because of the order of sequence in which the safety case must be established: defining the SoS of interest to analyze hazards in order to reduce risks.

For this reason, the MBSAF will consist of $3 \mathrm{~A} 3 \mathrm{AOs}$, two of which are safety related, see Figure 2. These A3AOs must match the content of meetings (see section 2.1) to maintain focus. The overview on system descriptions has already been researched by Borches. This study only addresses the overview on hazard analysis and risk reduction.

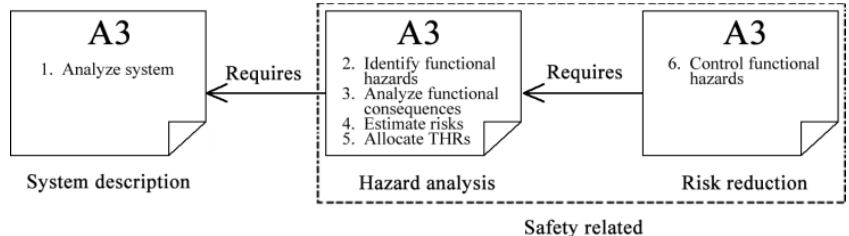

Figure 2. Allocation of A3AOs to meetings

Next step is to modify the A3AO tool so that these functions can be fulfilled. For this, we use the HSL-Zuid as a basis.

\subsection{HSL-Zuid A3AO hazard analysis}

Function 2 and function 3 of the $\mathrm{A} 3 \mathrm{AO}$ on hazard analysis contain functions, failure conditions and failure effects (also stated in Figure 1) making them automatically part of the functional view. For the HSL-Zuid, we start with a functional block diagram (FBD), because it can list functions, provide time-sequence and gives the possibility to include details on the interfaces between functions. A distinction between desired flow (normal scenario) and hazardous flow (failure scenario) is desirable as we want to indicate a hazard. To clarify the connections between function, flows and hazards, we include the FBD with flows, which results in a functional flow block diagram (FFBD) which also gives the possibility to cover function 3 . Function 4 and function 5 are key parameters that are indicated by numbers or classifications, which means they must be part of the quantification view. For the HSL-Zuid, we choose a matrix to list the risks and THRs. Figure 3 shows the 4 functions of the $\mathrm{A} 3 \mathrm{AO}$ on hazard analysis of the safety case of the HSL-Zuid with the allocation to views.

\subsection{HSL-Zuid A3AO risk reduction}

Figure 3 also shows the $\mathrm{A} 3 \mathrm{AO}$ on risk reduction only covers function 6 . This function contains barriers for prevention or correction. These barriers are tailored to the identified functional hazards from function 2 , which is shown as a feedback loop in Figure 3. Next to function 2, function 6 also needs input from function 5 , to determine which functions must be included or excluded. For the HSL-Zuid, we use a bow-tie diagram as a functional view, because it can list functions, failed functions, causes and consequences, and preventive and corrective barriers, but especially because it puts focus on creation of barriers instead of identifying hazards (as was the case with the FFBD). 


\subsection{HSL-Zuid Stakeholder requirements}

This study also addresses the design of the diagrams, views, and total A3AOs of the HSL-Zuid by implementation of user-centered requirements. These set out the need of all potential users by derivation of requirements from stakeholders through iterations of the A3AOs. These requirements enhance effectiveness and acceptation. According to Rajabalinejad [17], realization of stakeholders' values and their ranking can be a challenging task due to a high number of stakeholders and their competing or conflicting interest. This means that these requirements are not only case-specific, but also participant-specific. The requirements are shown in Table 1.

Table 1. Stakeholder requirements for A3AO safety case of the HSL-Zuid

\begin{tabular}{lll}
\hline \hline & Requirement description & Regarding \\
\hline 1 & $\begin{array}{l}\text { Show preconditions and } \\
\text { postconditions of the SoS }\end{array}$ & Hazard analysis \\
\hline 2 & $\begin{array}{l}\text { Show effects of failed } \\
\text { components on other components } \\
\text { because of stakeholder liability }\end{array}$ & $\begin{array}{l}\text { Hazard analysis } \\
+ \text { Risk reduction }\end{array}$ \\
\hline 3 & $\begin{array}{l}\text { Show allocation of hazards to } \\
\text { responsible components }\end{array}$ & $\begin{array}{l}\text { Hazard analysis } \\
+ \text { Risk reduction }\end{array}$ \\
\hline 4 & Show SoS total current risk status & $\begin{array}{l}\text { Hazard analysis } \\
+ \text { Risk reduction }\end{array}$ \\
\hline 5 & Show hazard control assessment & Risk reduction \\
\hline 6 & $\begin{array}{l}\text { Show emergency and } \\
\text { contingency arrangements }\end{array}$ & Risk reduction \\
\hline \hline
\end{tabular}

Stakeholder requirement \#1 is covered by making use of visual aids that give extra information about the preconditions of the first- and last function.

Stakeholder requirement \#2 indicates a need to get insight into the physical breakdown of the system to determine the relations between components to get insight into the responsibility of stakeholders. For the HSL-Zuid, we use a physical block diagram (PBD) to show relationships between components.

In early stages of this study, we found out that a stakeholder loses attention whenever it is not clear if the relevant hazard is under his/her responsibility, which is defined in stakeholder requirement \#3. For both functional views of the MBSAF of the HSL-Zuid, this can be achieved by making use of swimlanes, which are vertical or horizontal bands in the diagram that divide the diagram into logical areas or partitions, and -for the HSL-Zuid- make it possible to group functions to its responsible component. For the physical view and quantification view we choose to include the blocks, tables and graphs with the names of the stakeholders.

Stakeholder requirement \#4 about the SoS total current risk status can also be considered as a key parameter that can be included in the quantification view of both A3AOs. For the HSL-Zuid, we choose to present this in a table and graph.

Earlier, we stated the possibility of bow-tie diagrams to present barriers. This diagram also gives the possibility to cover stakeholder requirement $\# 5$, as the methodology provides a presentation for failed vs. broken barriers, but also to cover stakeholder requirement $\# 6$, as the methodology also provides a presentation for creation of barriers for discussing preventive (on the left-side) and corrective (on the right-side) elements.

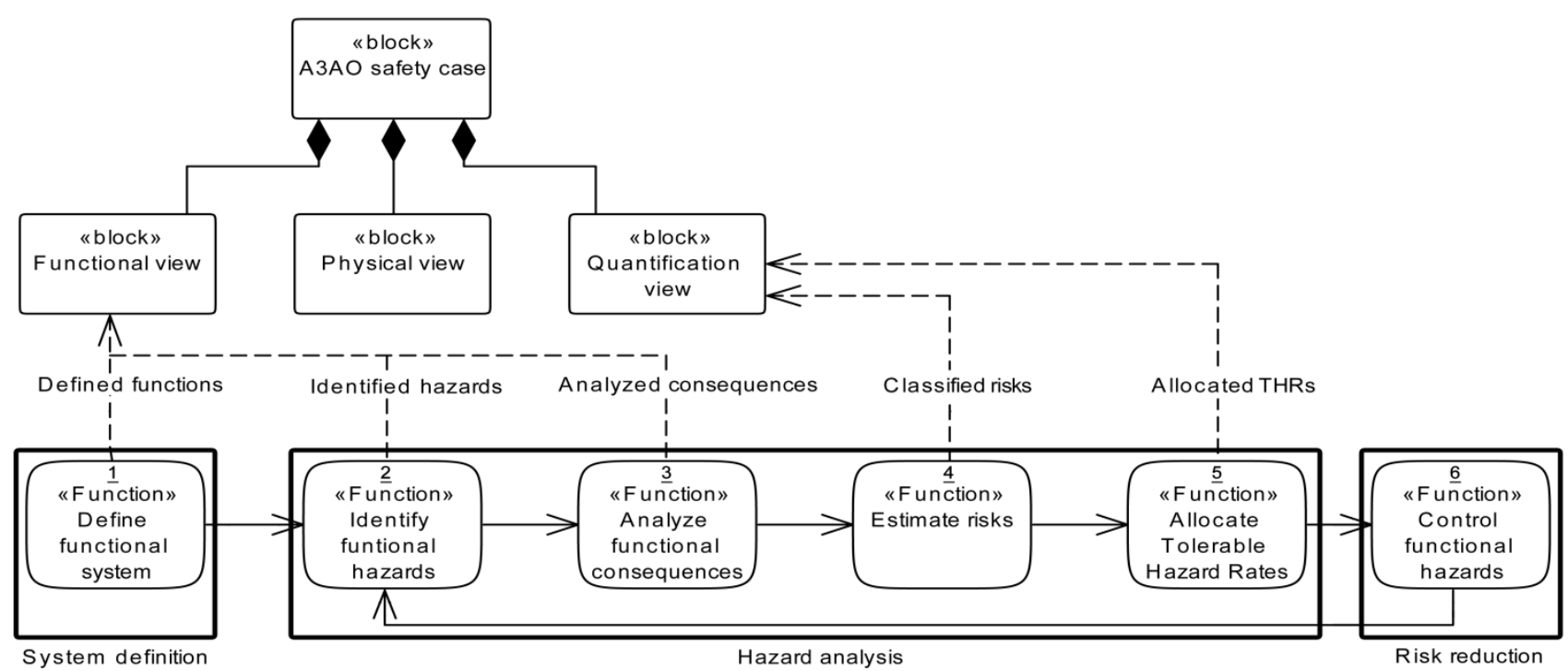

Figure 3. Functional interfaces in the MBSAF 


\subsection{MBSAF}

To capture the architectural knowledge of the HSL-Zuid, we set out guidelines, so that this MBSAF is reusable for similar safety cases.

At first, the 7 steps that are defined by Borches remain valid. These are: collect system concerns, create top level view, decompose top level view, quantify key parameters, complete A3 model, summary, share, adapt and store.

In contrast, these steps are no longer based on a system definition but on a safety case. This results in a change of goals and content of the three views in the A3AOs. The architectural knowledge of the $\mathrm{A} 3 \mathrm{AO}$ on hazard analysis can be captured by: create functional view with identified functional hazards and functional consequences, and create quantification view with estimated risks and allocated THRs. The A3AO architectural knowledge on risk reduction can be captured by: create functional view with controlled hazards.

With this in mind, the basis for capturing the architectural knowledge for both $\mathrm{A} 3 \mathrm{AO}$ s from which can be built on is shown by the two A3AOs in Figure 4, which are used for the HSL-Zuid and can be seen as an example of the A3 model for safety cases.

\section{Discussion}

Centralization of hazard analysis or risk reduction fosters focus, but subsequently also means that the A3AOs are always depending on each other and cannot be set up separately.

Next to this, the functions that are used for allocation to one of the three views are extracted from a common safety method used in Railway domain, which may reflect domain dependency of this framework. Likewise, the diagrams we used for the three views of the HSL-Zuid also differ per case and per stakeholder making this framework less suitable for a large target group. Another factor that involves dependency is the fact that the predominant view on both overviews is the functional view. This is highly related to the nature of the safety analysis. A Functional

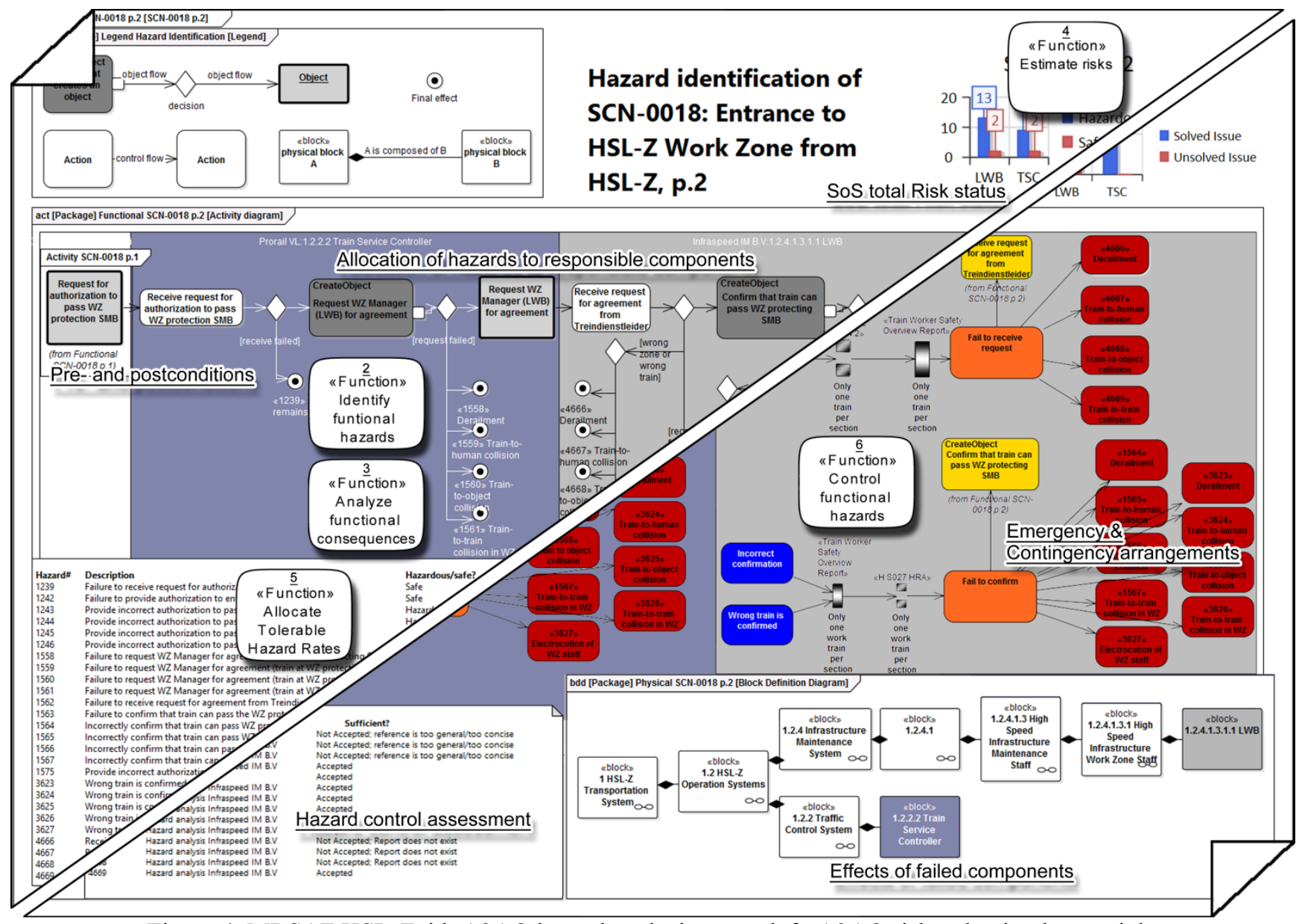

Figure 4. MBSAF HSL-Zuid: A3AO hazard analysis upper-left, A3AO risk reduction lower-right 
Hazard Analysis is based -as the name suggests- on functional behavior. A safety analysis focusing more on physical behavior would likely result in another predominant view of the A3AOs.

\section{Conclusion}

Given the shift to transparency and the rising demands of the general public for safety, there is a need to demonstrate the safety level of the system by using the safety case approach.

Here, we propose a model-based safety architecture framework (MBSAF) that centralizes hazard analysis and risk reduction in separate overviews to foster focus and avoids idleness of stakeholders, moving away from subjects that must be discussed to fulfill the stated purpose of the meeting and therefore; shortening the development cycle in safety case development.

If we compare this MBSAF with traditional documents, it enhances collaboration and communication and provides support for managing system complexity and system overview.

Nevertheless, the extraction of functions from safety standards used in Railway domain entails domain dependability for this framework. Taking into account other safety analyses that can be used to develop a safety case would more likely result in a more generic MBSAF.

Next to domain-dependency, although user-requirements increase acceptation and effectiveness of the architecture framework, these requirements differ per safety case.

The MBSAF captures and shares architectural knowledge of a safety case in A3AOs which results in increasing and effective interaction between stakeholders, more overview for managing safety complexity, more insight into finding required safety information, and therefore; an increasing efficiency in safety engineering.

We plan to use this framework and apply it to other SoS safety cases.

\section{References}

[1] P. D. Borches, A3 Architecture Overviews, Doctoral Thesis, Department of Engineering Technology (CTW), University of Twente, Enschede, The Netherlands, 2010.

[2] C. Piaszczyk, "Model Based Systems Engineering with Department of Defense Architectural Framework," Systems Engineering, Vol 14, No. 3, pp. 305-326, Feb. 2011.

[3] Z. Chaczko, A. S. Kohli, R. Klempous and J. Nikodem, "Middleware Integration Model for Smart Hospital System Using the Open Group Architecture Framework (TOGAF)," Proc. IEEE $14^{\text {th }}$ International Conference on
Intelligent Engineering Systems (INES), Las Palmas, pp. 215-220, May 2010.

[4] P. Bikar, "An open architecture framework for safety and Security", Proc. IEEE International Conference on Communication Workshops, Dresden, pp. 1-5, June 2009.

[5] D. D. Black, M. E. C. Hull and K. Jackson, "Systems engineering and safety - a framework,", IET Software, Vol 5, No. 1, pp. 43-53, February 2011.

[6] K. Jamboti and P. Liggesmeyer, "A framework for generating integrated component fault trees from architectural views", Proc. IEEE $14^{\text {th }}$ International Symposium on High-Assurance Systems Engineering (HASE), Omaha, pp. 114-121, October 2012.

[7] J. -Y Park and Y. -W Park, "Model-based Concurrent Systems Design for Safety," Concurrent Engineering, Vol 12, No. 4, pp. 287-294, December 2004.

[8] V. Chiprianov, K. Falkner, L. Gallon and M. Munier, "Towards Modelling and Analysing Non-functional Properties of Systems of Systems," Proc. IEEE $9^{\text {th }}$ International Conference on System of Systems Engineering (SoSE), Adelade, pp. 289-294, June 2014.

[9] P. America, P. van de Laar and G. Muller, "Experiences in evolvability research," Advanced Engineering Informatics, Vol 26, No. 3, pp. 478-486, August 2012.

[10] B. Wiulsrød, G. Muller and M. Penotti, "Architecting Diesel Engine Control System using A3 Architecture Overview," Proc. $22^{\text {nd }}$ annual international Symposium of the International Council on Systems Engineering (INCOSE), Rome, pp. 2429-2443, 2012.

[11] J. Holt and S. Perry, SysML for Systems Engineering, IET, 2008.

[12] S.C. Cook, "Towards designing innovative SoSE approaches for the Australian defence force," Proc. IEEE $9^{\text {th }}$ International Conference on System of Systems Engineering (SoSE), Adelade, pp. 295-300, June 2014.

[13] J. Zachman, "A framework for information systems architecture," IBM Systems Journal, Vol 26, No. 3, 1987.

[14] J. E. Cockshott, "Probability bow-ties: A Transparent Risk Management Tool," Process Safety and Environmental Protection, Vol 83, No. 4, pp. 307-316, July 2005.

[15] F. Aqlan and E.M. Ali, "Integrating lean principles and fuzzy bow-tie analysis for risk assessment in chemical industry," Journal of Loss Prevention in the Process Industries, Vol 29, pp. 39-48, May 2014.

[16] B. S. Blanchard and W. J. Fabrycky, Systems Engineering and Analysis, pp. 51, Pearson, Boston, 2011.

[17] M. Rajabalinejad and G.M. Bonnema, "Determination of stakeholders' consensus over values of system of systems," Proc. IEEE $9^{\text {th }}$ International Conference on System of Systems Engineering (SoSE), Adelade, pp. 2530, June 2014. 